\title{
A Case of Secretory Carcinoma That Occurred in the Buccal Submucosa
}

\author{
Saito Yoshiro1,2, Kurasawa Yuya, ${ }^{1,2}$, Moriya Takashi',2, Ikeda Kenitiro, ${ }^{1,2,3}$, \\ Kushihashi Yukiomi 1,2,3, Egawa Shunya ${ }^{1,2,3}$, Katsuta Hideyuki ${ }^{1,2,3}$, Shimane Toshikazu ${ }^{1,2,3}$ \\ ${ }^{1}$ Head and Neck Oncology Center, Showa University, Tokyo, Japan \\ ${ }^{2}$ Department of Oral and Maxillofacial Surgery, Division of Oral Oncology, Showa University, School of Dentistry, Tokyo, Japan \\ ${ }^{3}$ Department of Otorhinolaryngology, Showa University School of Medicine, Tokyo, Japan \\ Email: y.saito62@dent.showa-u.ac.jp
}

How to cite this paper: Yoshiro, S., Yuya, K., Takashi, M., Kenitiro, I., Yukiomi, K., Shunya, E., Hideyuki, K. and Toshikazu, S. (2019) A Case of Secretory Carcinoma That Occurred in the Buccal Submucosa. International Journal of Otolaryngology and Head \& Neck Surgery, 8, 191-197.

https://doi.org/10.4236/ijohns.2019.86021

Received: September 16, 2019

Accepted: October 21, 2019

Published: October 24, 2019

Copyright $\odot 2019$ by author(s) and Scientific Research Publishing Inc. This work is licensed under the Creative Commons Attribution International License (CC BY 4.0).

http://creativecommons.org/licenses/by/4.0/

\begin{abstract}
Secretory carcinoma (SC) is a malignant salivary gland tumor that has been first reported by Skalova et al. in 2010. Histologically, it shows solidity infiltrated with very small cystic cavities and cribriform and papillary features and includes periodic acid-Schiff stain-positive, acid-fast secretions. The cells have oval nuclei, and vacuolated cytoplasm and foamy secretions are seen. Anaplasia is not strong and mitotic figures are rarely seen. These features closely resemble AciCC. Immunohistologically, it is thought to be positive for S-100 protein, vimentin, and mammaglobin and negative for DOG1. The presence of the ETV6-NTRK 3 fusion gene is essential in diagnosing secretory carcinoma. In this report, we describe a case of SC in a 52-year-old woman. She was referred to our center because of a mass in left buccal mucosa. A soft and elastic submucosal mass measuring approximately $10 \mathrm{~mm} \times 10 \mathrm{~mm}$ in size with a smooth surface was seen in the buccal mucosa in an area corresponding to the left mandibular canine to premolars. The imaging findings revealed that a high-intensity lesion was seen on T2-weighted images. Immunohistochemicalstaing for S-100 protein and vimentin were positive. Furthermore, genetic examination detected the presence of the ETV6-NTRK3 fusion gene. Based on these findings, the definitive diagnosis was secretory carcinoma.
\end{abstract}

\section{Keywords}

Secretory Carcinoma, ETV6-NTRK3 Fusion Gene, Buccal Mucosa

\section{Introduction}

Mammary analogue secretory carcinoma (MASC) is a malignant salivary gland 
tumor that was first reported by Skalova et al. [1] in 2010. Histopathologically, it features a histological image reminiscent of secretory carcinoma of the breast, while also harboring the ETV6-NTRK3 fusion gene. Because there were previous patients with the ETV6-NTRK3 fusion gene among tumors classified as a subtype of acinic cell carcinoma (AciCC), it came to be labeled as secretory carcinoma in the 2017 World Health Organization (WHO) classification. As it has been a recently established disease entity, there are few reports. Moreover, secretory carcinomas are thought to occur mostly in the parotid gland in previous reports; occurrence in the minor salivary glands is considered to be rare [2]. We herein report a case of secretory carcinoma that occurred in the buccal submucosa, together with a discussion of the literature.

\section{Case}

A 52-year-old woman had felt a mass in her left buccal mucosa since 3 months. However, she took no action as there was no pain. When receiving dental treatment by hergeneral dental practitioner, she was told it was a lesion and was referred to our center. She had a history of uterine myoma, hyperlipidemia, and diabetes (hemoglobin A1c 6.5\%). A soft and elastic submucosal mass measuring approximately $10 \mathrm{~mm} \times 10 \mathrm{~mm}$ in size with a smooth surface was seen in the buccal mucosa in an area corresponding to the left mandibular canine to premolars (Figure 1). The mass had poor mobility and no tenderness was noted. Yellow lipoma-like lesions were seen in the anterior and posterior portions of the mass. Enlarged lymph nodes were palpated in the right submandibular region. Delineation of the lesion from computed tomography (CT) findings was difficult due to metallic artifacts, but an enlarged lymph node measuring $15 \mathrm{~mm}$ $\times 10 \mathrm{~mm}$ in size was seen in the left submandibular region. Inmagnetic resonance imaging (MRI) findings, a high-intensity lesion measuring $10 \mathrm{~mm} \times 9.5$ $\mathrm{mm} \times 6 \mathrm{~mm}$ in size was seen in the left buccal region on T2-weighted images (Figure 2). No obvious capsular structure was seen, the deep portion was in

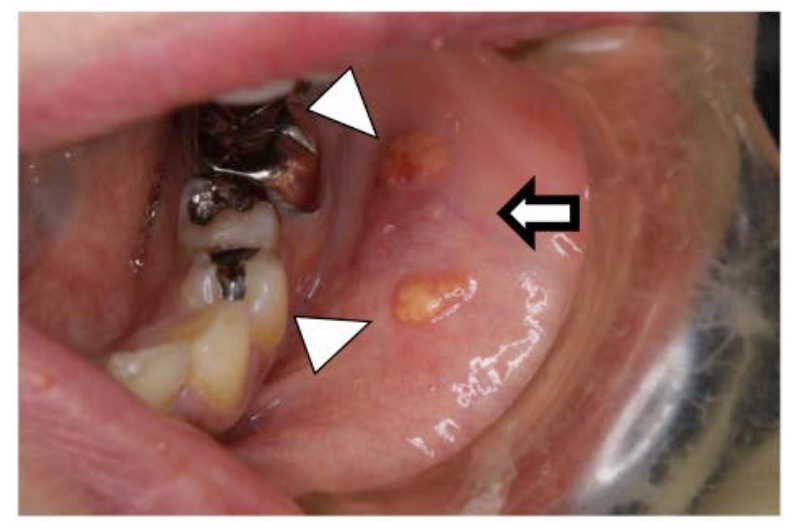

Figure 1. Oral findings. A soft, elastic submucosal mass measuring approximately $10 \mathrm{~mm}$ $\times 10 \mathrm{~mm}$ in size with a smooth surface is seen in the buccal mucosa in an area corresponding to the left mandibular canine to premolars (arrow). Yellow lipoma-like lesions are seen in the anterior and posterior portions of the mass (arrowhead). 


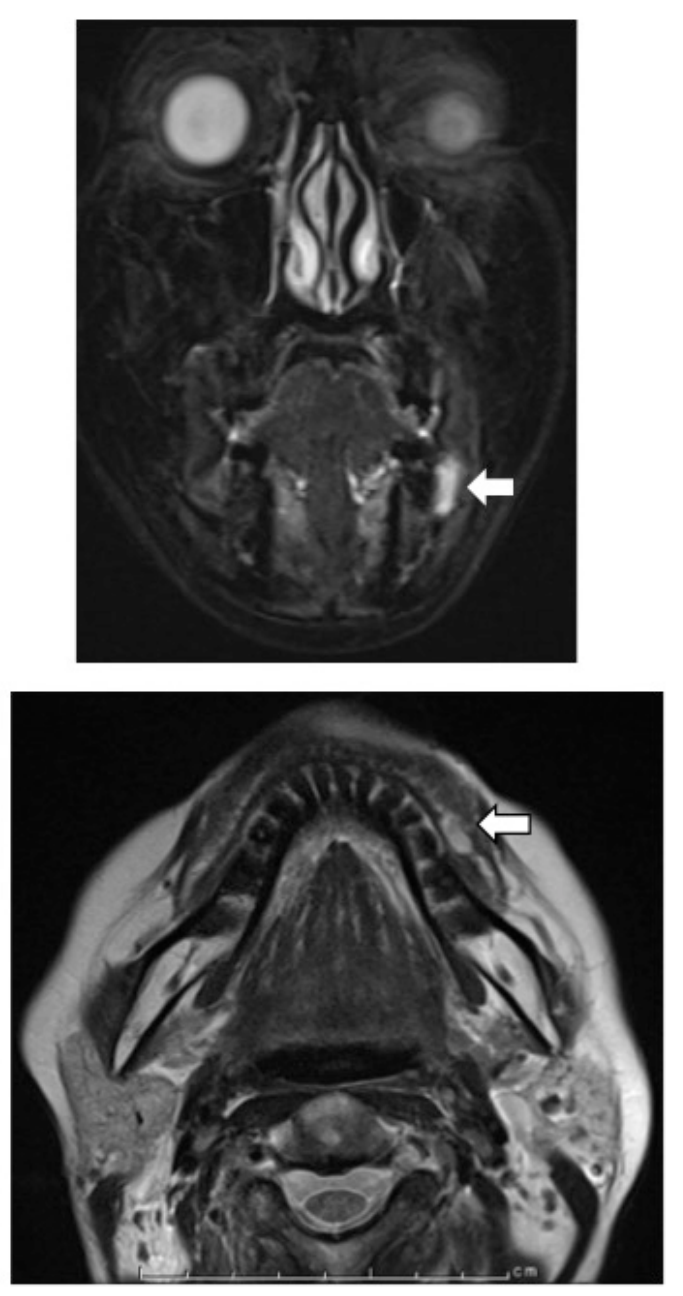

Figure 2. MRI findings. A high-intensity lesion measuring $10 \mathrm{~mm} \times 9.5 \mathrm{~mm} \times 6 \mathrm{~mm}$ in size is seen in the left buccal region on T2-weighted images (arrow).

contact with the buccinator, and the border was rather indistinct. Thus, invasion was a possibility and the findings were suggestive of a minor salivary gland malignancy. Clinically, it was diagnosed as a left buccal submucosal salivary gland malignancy.

\section{Treatment and Course}

Following fine needle aspiration biopsy of the enlarged lymph node in the left submandibular region, the tumor was diagnosed as Class II. Clinically, however, metastasis could not be excluded and we proposed resection of the left buccal submucosal malignancy and neck dissection. However, the patient refused consent for the neck dissection. Therefore, with the promise of rigorous postoperative follow-up, resection of the left buccal mucosal malignancy was performed under general anesthesia in October 2016. The resection was performed along with a sufficient margin of safety around the tumor, including the lipoma-like lesions. The tumor was resected, and after partial plication anteroposteriorly the low surface was covered with a polyglycolic acid sheet and plasma derivative. 
The resected specimen measured $30 \times 30 \times 10 \mathrm{~mm}$ in size, and a soft and elastic mass measuring $10 \mathrm{~mm} \times 10 \mathrm{~mm}$ in size was seen in the central submucosa. The histopathological findings showed existing small salivary gland tissue was seen from the submucosal layer to within the muscle layer multifocal nodal lesions similar to the replacing replaced salivary gland lobules. There was adhesion of epithelial components with glandular cavity structures in the interior of the nodular lesions, consisting of sites made up of cribriform structures and partially solidregions. Immunohistologically, a majority of the cancer cells were positive for Cytokeratin AE1/AE3 (CKAE1/AE3), Cytokeratin CAM5.2 (CAM5.2), Cytokeratin 19 (CK19), S-100 protein, and mammaglobin and negative for anti-smooth muscle antibody, calponin, carcinoembryonic antigen, human epidermal growth factor receptor 2, and discovered on gastrointestinal stromal tumor 1 (DOG1) (Figure 3). Secretory carcinoma was suspected from pathological and immunohistological characteristics, and the ETV6-NTRK3 fusion gene was detected in a gene search. Based on the above findings, the definitive diagnosis was secretory carcinoma. The patient was followed and at 9 months postoperatively there was further enlargement of the left submandibular lymph nodes, which had been enlarged from before the surgery. Fine needle aspiration biopsy led to a diagnosis of Class III, and lymph node metastasis of the secretory carcinoma was suspected. A left neck dissection (Level 1-V) was performed in June 2017. Metastasis was seen in a submandibular lymph node, and
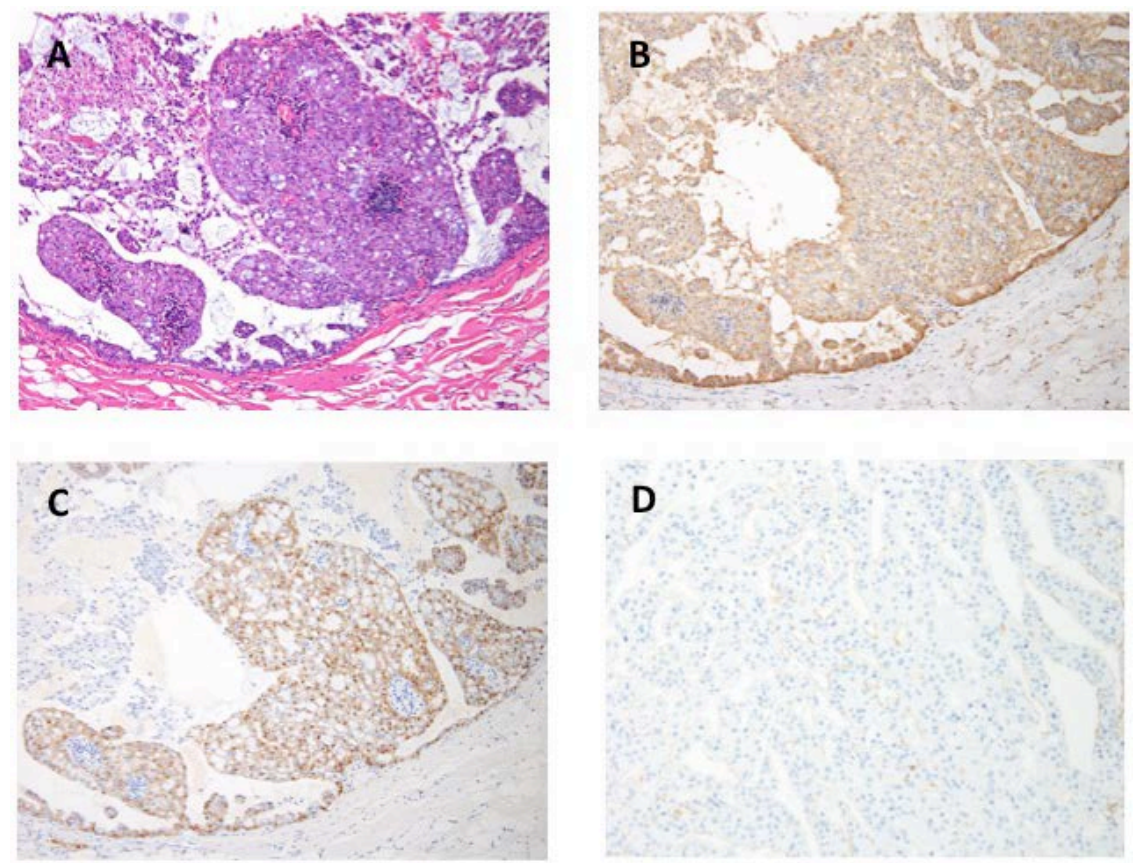

Figure 3. Histopathological and immunohistological findings. (A) Multifocal nodular lesions are seen similar to replaced the salivary gland lobules. Epithelial components with glandular cavity structures adhere in the interior of these lesions, which consist of sites made up of cribriform structures and some solid regions (HE stain); (B) Representative image of a mammaglobin-positive area; (C) Representative image of an S-100 protein-positive area; (D) Representative image of a DOG1-negative area. 
the histopathological findings showed a histological presentation similar to left buccal mucosal secretory carcinoma. Cervical lymph node metastasis secondary to secretory carcinoma was diagnosed. No recurrence has been seen for 1 year and 6 months postoperatively.

\section{Discussion}

MASC is a salivary gland malignancy for which the disease concept has been recently established. In 2002, Hirokawa et al. [3] reported that it is histologically a subtype of breast cancer that produces mammary gland secretions and resembles secretory carcinoma of the breast, a low-grade adenocarcinoma that presents varied images. Its morphology and immunostaining characteristics resemble some acinic cell carcinomas (AciCC). In 2010, Skalova et al. discovered that the ETV6-NTRK3 fusion gene is seen in both secretory carcinomas of the breast and AciCC with similar characteristics, and they proposed MASC as a novel disease. MASC was newly classified as a secretory carcinoma in the 2017 WHO classification of salivary gland tumors ( $4^{\text {th }}$ edition).

The gross and histopathological characteristics of secretory carcinoma are that most of the tumor consists of a solid portion, while occasionally large cystic cavities also form. Histologically, it shows solidity infiltrated with very small cystic cavities and cribriform and papillary features and includes periodic acid-Schiff stain-positive, acid-fast secretions. The cells have oval nuclei, and vacuolated cytoplasm and foamy secretions are seen. Anaplasia is not strong and mitotic figures are rarely seen [4]. These features closely resemble AciCC. Immunohistologically, it is thought to be positive for S-100 protein [1] [4] [5] [6], vimentin, [1] [4] [6] [7] and mammaglobin [1] [4] [5] [8] and negative for DOG1 [9] [10]. Nermine et al. [11] reported that all 9 patients with AciCC were negative for DOG1. Thus, S-100 protein, mammaglobin, and DOG1 are considered to be very useful in differentiating MASC from other salivary gland malignancies, including secretory carcinoma and AciCC. Recently, it has been reported that the ETV6-NTRK3 fusion gene has not been detected in any salivary gland tumors other than secretory carcinoma. The presence of the ETV6-NTRK3 fusion gene is essential in diagnosing secretory carcinoma [12] [13]. In the present case, multifocal modular lesions were seen histopathologically, as if replacing the lobules of the salivary glands. Inside these lesions, adhesion of epithelial components with glandular cavity structures, consisting of sites made up of cribriform structures and partially solid regions were seen. This is a finding that markedly resembles AciCC. Immunohistologically, the tumor was positive for mammaglobin and S-100 protein and negative for DOG1. Hence, secretory carcinoma was strongly suspected and the ETV6-NTRK3 fusion gene was positive. The patient was finally diagnosed with left buccal submucosal secretory carcinoma.

Sethi et al. [2] analyzed 92 patients with secretory carcinoma and reported their clinical features. The female to male ratio is about 1.2:1 and the mean age was 44.2 years. The tumors often grow painlessly. The primary site of onset is the parotid gland $(70 \%)$, followed by the submandibular gland (7\%). Occurrence 
in the minor salivary glands in the soft palate, buccal mucosa, base of the tongue, and lips has been previously reported. Chiosea et al. [5] reported cervical lymph node metastasis from secretory carcinoma in $22 \%$ of cases (four of 18 ), recurrence in three cases (three of 18), and distant metastasis in one case (one of 18). Although malignancy is somewhat higher in secretory carcinoma than in AciCC, no significant difference is seen between the two diseases in the disease-free survival rate and treatment success rate. Secretory carcinoma is difficult to diagnose clinically and cytologically, and when a low-grade malignancy such as AciCC is suspected clinically, it is important to consider secretory carcinoma in consultation with a pathologist when making a diagnosis. Cervical lymph node metastasis is also somewhat more common in secretory carcinoma than in AciCC and was seen in our patient. Thus, a careful examination for cervical lymph node metastasis and subsequent neck dissection without delay in metastasis cases are thought to be important. Although secretory carcinoma is generally considered to be a low-grade malignancy, sarcomatoid changes and poor outcomes are seen in some cases and careful examination and follow up is needed [14].

The disease concept of secretory carcinoma has been recently established. Currently, the first-line treatment is surgical resection, similar to other salivary gland malignancies [15]. However, the anaplastic lymphoma kinase (ALK) inhibitor crizotinib has been reported to significantly reduce ETV6-NTRK3 gene fusion, and it is potentially effective in the treatment of secretory carcinoma [16]. It is necessary to accumulate further elucidation of the pathology of this malignancy in a greater number of patients.

\section{Conflicts of Interest}

The authors declare no conflicts of interest regarding the publication of this paper.

\section{References}

[1] Skalova, A., Vanecek, T., Sima, R., et al. (2010) Mammary Analogue Secretory Carcinoma of Salivary Glands, Containing the ETV6-NTRK3 Fugson Gene: A Hitherto Undescribed Salivary Glands Tumor Entity. The American Journal of Surgical Pathology, 34, 599-680.

[2] Sethi, R., Kozin, E., et al. (2014) Mammary Analogue Secretory Carcinoma: Update on a New Diagnosis of Salivary Gland Malignancy. The Laryngoscope, 124, 188-195. https://doi.org/10.1002/lary.24254

[3] Hirokawa, M., Sugihata, K., Sai, T., et al. (2002) Secretory Carcinoma of the Breast: A Tumor Analogous to Salivary Gland Acinic Cell Carcinoma? Histopathology, 40, 223-229. https://doi.org/10.1046/j.1365-2559.2002.01346.x

[4] Skalova, A. (2013) Mammary Analogue Secretory Carcinoma of Salivary Glands Origin: An Update and Expanded Morphologic and Immunohistochemical Spectrum of Recently Described Entity. Head and Neck Pathology, 7, 30-36. https://doi.org/10.1007/s12105-013-0455-y

[5] Chisea, S.I., Griffth, C., Assaad, A. and Seethala, R.R. (2012) Clinicopathological Characterization of Mammary Analogue Secretory Carcinoma of Salivary Gland. 
Histopathrogy, 61, 387-394. https://doi.org/10.1111/j.1365-2559.2012.04232.x

[6] Petterson, F., Lian, D., Chau, P.Y. and Yan, B. (2012) Mammary Analogue Secretory Carcinoma: The First Submandibular Case Reported Including Findings on Fine Needle Aspiration Cytology. Head and Neck Pathology, 6, 135-139. https://doi.org/10.1007/s12105-011-0283-x

[7] Ito, S., Ishida, E., et al. (2012) Case Report of Mammary Analogue Secretory Carcinoma of the Parotid Gland. Pathology International, 62, 149-152. https://doi.org/10.1111/j.1440-1827.2011.02759.x

[8] Fehr, A., Loning, T. and Stenman, G. (2012) Mammary Analogue Secretory Carcinoma of the Salivary Glands with ETV6-NTR3 Gene Fusion. The American Journal of Surgical Pathology, 35, 1600-1602. https://doi.org/10.1097/PAS.0b013e31822832c7

[9] Jung, M.J., Song, J.S., et al. (2013) Finding and Characterizeing Mammary Analogue Secretoy Carcinoma of the Salivary Gland. Korean Journal of Pathology, 47, 36-43. https://doi.org/10.4132/KoreanJPathol.2013.47.1.36

[10] Majewska, H., Skalova, A., et al. (2015) Mammary Analogue Secretory Carcinoma of Salivary Glands: A New Entity Associated with ETV6 Gene Rearrangent. Virchows Archiv, 466, 245-254. https://doi.org/10.1007/s00428-014-1701-8

[11] Nermine, M.A.R. and Sarah, A.H. (2015) Diagnostic Role of DOG1 and p63 Immunohistochemistry in Salivary Gland Carcinomas. International Journal of Clinical and Experimental, 8, 9214-9222.

[12] Connor, A., Perez, O.B., Shago, M., Skálová, A. and Weinreb, I. (2012) Mamamry Analogue Secretory Carcinoma of Salivary Gland Origin with the ETV6 Gene Rearrangement by FISH: Expanded Morphologic and Immunochemical Spectrum of a Recently Described Entity. The American Journal of Surgical Pathology, 36, 27-34. https://doi.org/10.1097/PAS.0b013e318231542a

[13] Bishop, J.A. (2013) Uumasking MASC: Bringing to Light the Unique Morphologic, Immunestochemical and Genetic Features of the Newly Recognized Mammary Analogue Secretory Carcinoma of Salivary Glands. Head and Neck Pathology, 7, 35-39. https://doi.org/10.1007/s12105-013-0429-0

[14] Noda, T., Yamada, K., et al. (2015) A Case of Mammary Analogue Secretory Carcinoma with Salcomatoid Change. Stomatopharyngology, 28, 137-141.

[15] Ishi, K., Nakamatsu, K., et al. (2015) A Case of Mammary Analogue Secretory Carcinoma Arising in the Submandibular Region. Journal of Oral and Maxillofacial Surgery, 61, 564-569.

[16] MIkko, T., Iina, K., et al. (2013) Chaperones as Thermodynamic Sensors of Drug-Target Interactions Reveal Kinase Inhibitor Specificities in Living Cells. Nature Biotechnology, 31, 630-637. 